\title{
COMPARISON OF ENTERPRISE MODELLING METHODOLOGIES
}

\author{
K. KOSANKE \\ CIMOSA ASSOCIATION E.V. \\ STOCKHOLMER STR. 5, D-71034 BÖBLINGEN, GERMANY \\ TEL. +4970312776 65, FAX: +4970312766 98, \\ E-MAIL: KOSANKE@IPA.FHG.DE
}

\begin{abstract}
Business re-engineering and enterprise integration efforts are supported very efficiently by enterprise modelling methodologies. However, with the number of methodologies available the comparison and selection of the most suited one becomes a rather difficult task. Most modelling methodologies orient themselves on the life-cycle concept but usually cover different part of the cycle itself. In addition, terminology and modelling constructs/language for representation of the model contents are further obstacles to be overcome in the selection process.

Representation of modelling methodologies as business processes enables their comparability in terms of enterprise life-cycle coverage and capability of enterprise information collection and representation. The paper presents the results of an analysis carried out for several enterprise modelling methodologies highlighting their similarities and differences. All modelling methodologies follow the enterprise life-cycle with emphasis on the requirements definition phase. Several methodologies carry enterprise modelling through design specification and implementation description to operation and model maintenance. Language expressiveness is quite different both in number of language constructs provided and their use in enterprise modelling.

In addition, the business process representation provides explicit identification of the information to be collected in the model. Both the information needed for the different modelling tasks and the results of the tasks can be explicitly identified thereby guiding the user of the methodology.

The analysis identifies the compatibilities of the different enterprise modelling methodologies and their emphasis on particular parts of the enterprise modelling task. It is hoped that this work also helps to harmonise the results of enterprise modelling as well as the terminology used. Both are very much needed in the work on enterprise integration.
\end{abstract}

Keywords

Enterprise Integration, Enterprise Architectures, Enterprise Modelling, Business Modelling, Modelling Methodologies, Modelling Languages.

\section{INTRODUCTION}

Methodology is the system of methods and principles used in a particular discipline. Method is a way of proceeding or doing something; the technique or arrangement of work for a particular field ${ }^{1}$.

1 Collins Dictionary and Thesaurus, 1987 
These definitions imply the process nature of both methods and methodology. Process representations, especially graphical ones, are much more easily understood and comparable with each other. In addition, all of such methodologies are based on the life-cycle concept which allows a comparison of the different methods in terms of the coverage of different process steps in the lifecycle. The paper presents examples of the process representation of several Enterprise Modelling Methodologies. The graphical representation of the different methodologies as process models is based on CIMOSA an ESPRIT supported development.

The paper is intended to demonstrate the benefits of a common process oriented representation of modelling methodologies. It does not claim completeness and full correctness of the process models, which will need further work to capture all the details of the textual description available today.

The different methodologies represented and compared are ARIS $^{2}$, CIMOSA $^{3}$, GRAI/GIM ${ }^{4}$, IEM $^{5}$ and PERA ${ }^{6}$ with process models currently available only for CIMOSA, IEM and PERA. The work is based on material describing the different methodologies available to the author. It represents the authors view of the methodologies and may be modified in the course of further discussions with the developers and owners of the methodologies themselves. Due to the limitations of a paper only the example of the modelling methodologies with the widest life-cycle coverage (PERA) is presented with the graphical representation of its process model.

In addition, the paper compares the modelling languages used in the different methodologies. For more information on CIMOSA representation see references [1] and [2]. For a comparison of different methodologies see also references [3] and [4].

\section{THE METHODOLOGIES - AN OVERVIEW}

The different modelling methodologies have all been developed with different applications in mind. Therefore emphasis is on different aspects of enterprise modelling. Nevertheless they all contribute to enterprise integration and therefore should contribute to a common view on the subject. This paper tries to highlight the differences in goal and application areas of the different methodologies.

\section{ARIS (ARchitecture for Information Systems) [5]}

The ARIS focus is on the design of enterprise information systems. Therefore it provides specific modelling support for the Information Technology part of the enterprise (IT concept support). ARIS supports enterprise modelling from operation concept and IT concept to IT system implementation.

\section{CIMOSA (CIM Open Systems Architecture) [1][2]}

CIMOSA models are intended to be used for operational support rather than as project guides in developing or re-engineering business entities. Operational use is understood as decision support for evaluating operational alternatives as well as model driven operation control and monitoring. CIMOSA supports the engineering of enterprise models from requirements definition to implementation description, their operational use and model maintenance supporting system changes and business re-engineering.

\section{GRAI/GIM (Graphs with Results and Activities Interrelated/GRAI Integrated Methodology) [6]}

GRAI was initially developed to model the decisional structure of a manufacturing enterprise for strategic, tactical and operational planning. GRAI was extended to support the design of CIM systems leading to GIM as an integrated methodology for business process modelling. With special emphasis on the decisional aspects, the concept (analysis), structure (user oriented design) and realisation (technical oriented design) phases of the life-cycle concept are supported.

ARchitectur für Informations Systeme (Architecture for Information Systems)

Open System Architecture for CIM

Graphe à Résultant et Activités Interreliés(Graphs with Results and Activities Interrelated)/GRAI Integrated Methodology

Integrated Enterprise Modelling

Purdue Enterprise Reference Architecture 
IEM (Integrated Enterprise Modelling) [4][10]

The IEM modelling methodology supports creation of enterprise models for business reengineering and therefore allows also to model process dynamics for evaluation of operational alternatives. IEM supports the main phases of the enterprise life-cycle (requirements, design, implementation and model up-date).

\section{PERA (Purdue Enterprise Reference Architecture) [7]}

The PERA modelling methodology is intended to support and guide the development of the Master Plan for an Enterprise Business Entity. The methodology covers the complete project of introduction, implementation and operation of an enterprise business entity which may be either part of a larger entity or be the complete enterprise itself. The life-cycle starts with the definition of the Business Entity to be modelled, identifying its mission, vision, management philosophy, mandates, defines project sponsors, leaders and members, etc. and ends with obsolescence of the plant at the end of the operational phase.

\section{PROCESS MODELS OF MODELLING METHODOLOGIES}

The modelling methodologies are described in terms of their information exchange with the environment (CIMOSA Domains) and their internal process structure. The different processes (DP $=$ Domain Process) identified correspond to the phases of the system life-cycle. These processes are further detailed as either sub-processes $(\mathrm{BP}=$ Business Process) or activities (EA $=$ Enterprise Activity). Behavioural Rules define the process flow (control flow) identifying the conditions for continuation after ending an activity. Due to the space constraints of the paper the process model of only one of the methodologies (PERA) is presented (Figures 1 to 3). The information used and produced in the different activities is presented in Table 1 . This part of enterprise modelling allows to identify and provide/eliminate missing or redundant information and no value information, respectively. A comparison of the different methodologies (PERA, CIMOSA and IEM) is presented in Table 2 (at the end of the paper). The CIMOSA modelling methodology is described in a recent publication [8].

\section{Process Model of PERA (Purdue Enterprise Reference Architecture)}

The PERA modelling methodology covers the complete enterprise life-cycle starting from Business Entity Identification and ending with the turn-down of the plant at the end of the operational phase. Its life-cycle phases are described for personnel, information and product operational requirements leading to an information architecture, a human and organisational architecture and a manufacturing equipment architecture.

\section{Process Representation of the PERA Modelling Methodology}

The following is an attempt to establish a process model of the Purdue Enterprise Reference Architecture methodology using the CIMOSA modelling language (constructs). A draft of the process model is provided which has been developed in co-operation with T.J. Williams and coworkers. The modelling environment overview (Figure 1) provides the relation between the further detailed CIMOSA Domain 'Enterprise Business Entity Master Plan Development' and the noneCIMOSA Domains. Information exchange is identified on a rather high level indicating information and events exchanged between the CIMOSA Domain and the none-CIMOSA Domains.

\section{PERA Process Model Overview}

The details of the CIMOSA Domain are shown in Figure 2. Seven Domain Process have been defined covering each one of the different phases of the system life-cycle identified in the layering diagram of the PERA methodology. Enterprise Events have been defined which enable the cooperation of the different Domain Processes indicating completion of processes or needs for changes of results of previous ones. Figure 3 provides an example of the details of the different Domain Processes represented on Business Processes and Enterprise Activity level. The example shows the parallel efforts for the three architectures of PERA for information, human and organisation and manufacturing equipment. Behavioural Rules are only indicated but are not further defined. 


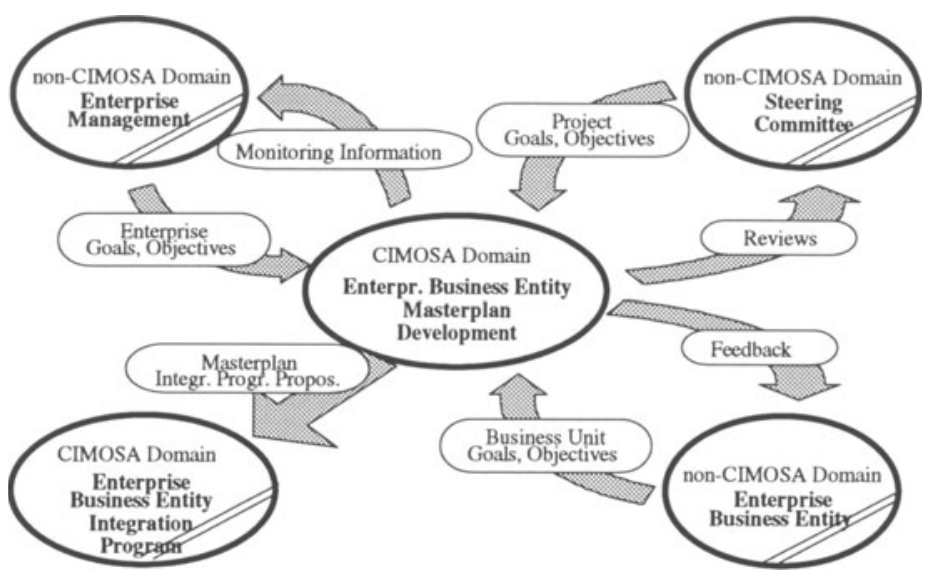

Figure 1 PERA Enterprise Business Entity Masterplan Development Project - Relation to other Domains

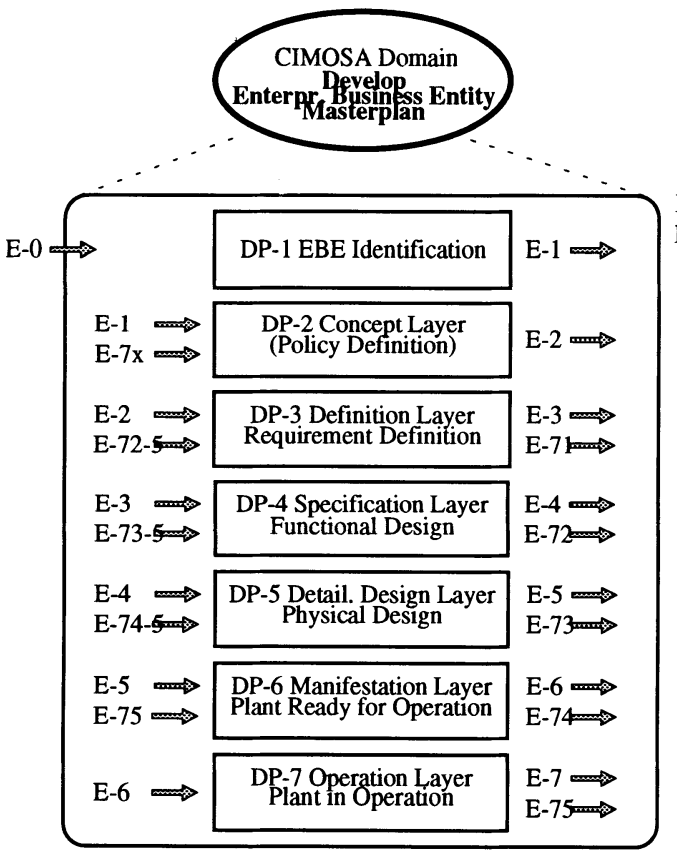

$\mathrm{DP}=$ Domain Process

$\mathrm{E}=$ Event

List of Events

E- $0=$ Initiate EBE Identification

E-1 = EBE Identification completed

E-2 = Concept Layer completed

E-3 = Definition Layer completed

E-4 = Specification Layer completed

E-5 = Detailed Design Layer completed

E-6 = Manifestation LAyer completed

$\mathrm{E}-7=$ Operation Layer completed

$\mathrm{E}-7 \mathrm{x}=$ Change Request (Domain internal)

Figure 2 PERA Enterprise Business Entity Masterplan Development Project - Domain Processes

\section{PERA Information Identification}

Representing the modelling methodology as a business process allows to identify the information used and produced by the different task. This can become the knowledge base of the enterprise ensuring a content which is identified as being both used and produced during enterprise operation. 
Table 1 shows an example of the information needed and created by the PERA methodology. The different information objects described in the PERA literature have been structured into a set of enterprise objects (CIMOSA term) which present a part of a high level information model for the PERA methodology. The tasks which use and produce the information objects are indicated. Referring to the PERA literature this table indicates the consistency problems of textual descriptions.

Several of the information objects identified in the PERA methodology are either not used or not produced. Completing this table according to the business process representation at the necessary level of detail allows to identify all information and therefore provides a complete and consistent information model of the enterprise information used and produced during the modelling process. Providing real time maintenance for such an enterprise model will ensure an always up-todate knowledge base of the enterprise.

Table 1 Information (Enterprise) Objects used and produced by the PERA Methodology

\begin{tabular}{|c|c|c|}
\hline Enterprise Object & Where Used ${ }^{7}$ & Where Produced ${ }^{7}$ \\
\hline \multicolumn{3}{|l|}{ Management Mission, Vision and Values } \\
\hline Company Business & EA-20,21 (FII-2-1) & DM 'Enterprise Management' \\
\hline Marketing Strategies & EA-22 (FII-2-1/II-3-4) & DM 'Enterprise Management' \\
\hline Enterprise Business Entity Information & EA-1,23 (FIII-1-1) & (FII-2-1) \\
\hline \multicolumn{3}{|l|}{ Goals and Objectives } \\
\hline Enterprise Level & EA-1 $\quad$ (FII-3-4) & DM 'Enterprise Management' \\
\hline Enterprise Business Entity Level & BP-3.1,3.2 (FI-2-1/2/8) & DM 'Enterpr. Business Entity' \\
\hline \multicolumn{3}{|l|}{ Internal Knowledge } \\
\hline Technology & EA-23,BP-3.2 & $\begin{array}{l}\text { DM 'Enterprise Management' } \\
\text { DM 'Enterpr. Business Entity' }\end{array}$ \\
\hline Mfg. Capabilities & (FI-2-1/2) & DM 'Enterprise Management' \\
\hline Operational Policies & (FI-2-2/8) & (FI-2-8/II-3-4) \\
\hline Mfg Requirements & DP-4 & (FII 3-4) \\
\hline \multicolumn{3}{|l|}{ Internal Constraints } \\
\hline Critical Success Factors & (FI-2-1/2) & DM 'Enterprise Management' \\
\hline \multicolumn{3}{|l|}{ External Knowledge } \\
\hline Technology & EA-23,BP-3.2 (FI-2-1) & DM 'Enterprise Management' \\
\hline Experience of other Industries & EA-23,BP-3.2 (FII-1-1) & \\
\hline User Requirements & DP-4 (FI-2-1) & DM 'Enterprise Management' \\
\hline Customer Requirements & (FI-2-2) & \\
\hline Legal Requirements & (FI-2-9) & \\
\hline Standards & (FI-2-8) & \\
\hline \multicolumn{3}{|l|}{ External Constraints } \\
\hline Economic Conditions & (FI-2-1/II-3-4) & \\
\hline World Markets & (FII-3-4) & \\
\hline Environment & (FII-3-4) & \\
\hline \multicolumn{3}{|l|}{ Planning Data } \\
\hline $\begin{array}{l}\text { Current State of Human and Mfg. } \\
\text { System. }\end{array}$ & (FI-2-1/8) & $(\mathrm{FI}-2-1 / 8)$ \\
\hline Future State of Human and Mfg. Syst. & $(\mathrm{FI}-2-1 / 8)$ & (FI-2-1/8) \\
\hline Transition Plan & $(\mathrm{FI}-2-1 / 8)$ & DP-2 (FI-2-1/8) \\
\hline New Plan & (FI-2-1) & DP-3 (FI-2-1) \\
\hline
\end{tabular}

7 Reference: DP/BP/EA (CIMOSA Process/Enterprise Activity) FI/II (PERA Figure) 


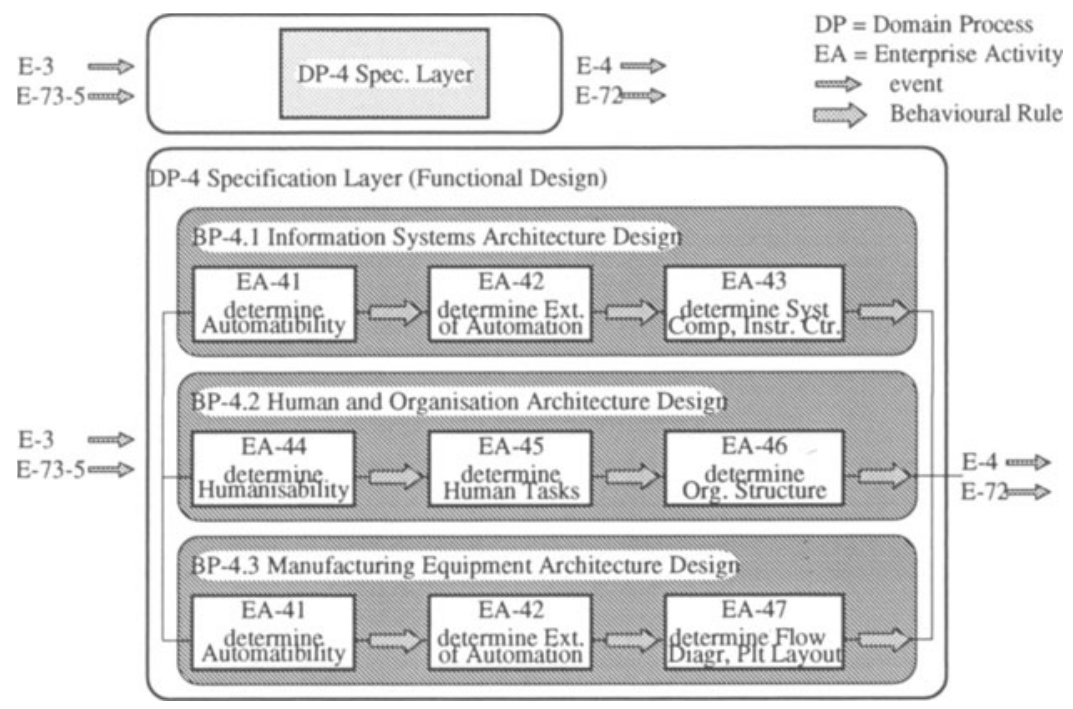

Figure 3 PERA Enterprise Business Entity Masterplan Development Project - Details of Domain Process DP-4 Specification Layer (Functional Design)

\section{Methodology Comparison PERA - CIMOSA - IEM}

Table 2 shows the process models of the three methodologies at the Business Process level with identification of lower level Enterprise Activities. The latter is still to be done for the IEM modelling methodology. The number of activities identified for PERA and CIMOSA are 48 and 77 respectively demonstrating the higher level of details provided by CIMOSA. This is needed for the intended use of the CIMOSA model.

The representation follows the system life-cycle concept identified for the PERA methodology adding the maintenance phase of CIMOSA and IEM. This comparison demonstrates the advantage of the process oriented presentation of the modelling methodologies enabling direct comparison of the different methods in terms of coverage of the system life-cycle and different emphasis on the different phases.

\section{MODELLING FRAMEWORK COMPARISON}

A more global comparison of all modelling methodologies identified in this paper is shown in Tables 3.1 to 3.3. Using the Generalised Enterprise Reference Architecture and Methodologies (GERAM) [9] definition of the life-cycle phases the corresponding parts of the different methodologies have been identified ${ }^{8}$. In addition to the life-cycle phases represented already in Table 2 for PERA, CIMOSA and IEM the Model Views and Genericity Levels are identified for the five methodologies investigated. The tables again indicate the terminology problem existing in enterprise modelling. But there is a surprisingly high level of terminology consistence.

\section{Life-cycle Dimension}

Table 3.1 indicates a rather similar coverage of the centre life-cycle phases (requirement, design, implementation) by all modelling methodologies. PERA covers the two uppermost GERAM layers

8 a 'not defined' entry means no formal identification exists. But the methodology may still provide specific solutions. 
for the identification of the Business Entity and definition of its management policies, etc. This information is assumed to be provided by enterprise management in all other methodologies.

The Operation Layer is explicitly defined in PERA only. Its existence in CIMOSA is recognised, but it is not seen as part of the modelling methodology. CIMOSA distinguishes between the enterprise engineering environment and the operation environment assuming models to be used as operational support (decision support tool) and directly in model driven operation control and monitoring. With this vision of enterprise model application, model maintenance is seen as a very important life-cycle phase, which is explicitly identified in both CIMOSA and IEM and contained in the operation layer of PERA.

The GRAI/GIM modelling framework distinguishes between the three architectural levels (Concept, Structure, Realisation) and three modelling activities (Analysis, User Oriented Design, Technical Oriented Design). The first two activities are relating to the first two architectural levels and the last activity is concerned with the realisation level. Two different sets of Model Views (see below) are identified for the different architectural levels.

\section{Model View Dimension}

Different views on the model help to reduce model complexity for the user. As shown in Table 3.2 such model views are provided by most methodologies, however, not all with the same capabilities. CIMOSA assumes one consistent enterprise model on which particular views are provided for the user in the engineering environment to allow for model engineering on a particular aspect of the enterprise operation (Function, Information Resource, Organisation, others tbd). ARIS provides a similar approach, but has identified the Control View for integrating the different views into a common process model. GRAI/GIM and PERA identify different views, but there is no real integration into one consistent model yet.

PERA changes its view concept across the life-cycle phases from a global view for the first and part of the second layer. It defines two views (Information Architecture and Manufacturing Architecture) for most of layer two and all of layer three. PERA continues thereafter with three views (Information Systems Architecture, Human \& Organisation Architecture, Manufacturing Equipment Architecture).

GRAI/GIM identifies a unique Decision View which is at the centre of the GRAI methodology enabling modelling of strategic, tactical and operational planning.

IEM does not defines model views explicitly but provides viewpoints on a common model. Therefor its modelling language constructs are related to the different views as well.

\section{Genericity Level Dimension}

This framework dimensions separates the particular model from the reference architecture which supports model creation. The reference architecture may contain generic building blocks or constructs for modelling (the words of the modelling language) and reference or partial models which may be used as macros in the modelling process. Except for PERA which only provides a single task module, all methodologies have a rather populated generic level and almost all provide sets of partial/reference models as well (Table 3.3).

\section{MODELLING LANGUAGE CONSTRUCTS COMPARISON}

A very extensive comparison between IEM and CIMOSA modelling constructs has been made jointly by the two originating teams in their efforts on trying to converge to a common modelling language. This comparison is described in a joined paper submitted to the European standardisation [10] which has lead to the ENV 12204 the pre-standard on enterprise modelling constructs [11].

Tables 4.1 and 4.2 give an overview of the modelling languages provided by the different modelling methodologies. In addition to GERAM, which does not define any language constructs, the ENV 12204 has been included as a reference. All methodologies provide some type of support for representation of the model contents. These languages consist of sets of generic constructs or building blocks to represent enterprise processes, activities, information, resources, organisation, etc. The constructs enable collection of relevant information allowing to describe the enterprise objects according to the modelling goal. Only PERA is not defining such modelling language but relies mainly on textual description of its methodology. 
The modelling constructs can be associated to model views even if the may play a role in other views as well. In Table 4.1 and 4.2 the construct sets are structured according to their major role in enterprise modelling.

\section{General Definitions}

Most methodologies provide some structuring definitions in addition to the specific constructs. These definitions identify either the model contents (GRAI/GIM, PERA) or distinguish between model engineering and model use (CIMOSA).

\section{Function View related}

Constructs for function representation are provided by all methodologies with specialisations provided by CIMOSA and IEM. Both provide the process representation in the function view as well. ARIS has defined the control view for the representation of its process chains. Both GRAI/GIM and PERA do not offer modelling of the dynamic behaviour of its processes.

\section{Decision View related}

This view is only provided by GRAI/GIM. It allows to model the decision structure of the enterprise as well as to differentiating between different types of decisions (strategic, tactical, operational) by identifying different time horizon for the decisions. All other methodologies model decision making activities as parts of their (management oriented) business processes.

\section{Information View related}

ARIS, CIMOSA and IEM all provide a rich set of constructs for information modelling. Both ARIS and CIMOSA include IT oriented modelling constructs for modelling the IT system. ARIS provides additional IT oriented modelling constructs in the control view and in the organisation view. GRAI/GIM has defined two modelling constructs for information modelling using the Entity Relationship Approach for representation of the information model.

\section{Resource View related}

Constructs for the resource view exist in CIMOSA and IEM. ARIS is concerned mainly with IT resources which are described in the control, information and organisation view. The construct technical resources is used to describe all non-IT resources.

\section{Organisation View related}

The organisation view is populated in ARIS, CIMOSA and IEM. Whereas in ARIS resource organisational aspects are included in this view, CIMOSA uses the organisation view for identification of organisational aspects only. The main purpose in CIMOSA is to identify responsibilities and authorisation on all other enterprise objects (processes, information, resources) and to establish an escape mechanism for out of line situations. IEM uses a special class of its Resource Object for identifying organisation entities.

Modelling Language Constructs Comparison ARIS - CIMOSA - GRAI/GIM - IEM- PERA Similar to the different aims of the different methods in terms of modelling results the expressiveness of the particular languages differ as well. Only CIMOSA has the vision of on an executable model for operation control and monitoring. Therefore its modelling language is a very expressive one. All other methodologies are focusing on particular situations from enterprise integration project descriptions (PERA), decision systems modelling and CIM system design (GRAI/GIM), information system design (ARIS) to business process re-engineering (IEM). Therefore their modelling languages are tuned to that particular application area resulting in more specialised constructs like ARIS (IT resource description), GRAI (decision view) and IEM (special object classes: Product, Order, Resource). On the other hand PERA is relying on textual description of its methodology providing only a construct for representation of task and its information inputs and outputs. Hopefully this comparison will result in more harmonisation of modelling languages both in their contents and their terminology. 


\section{SUMMARY}

The analysis demonstrates the value of process oriented representation of modelling methodologies. It provides comparability far beyond textual description in terms of coverage of the modelling processes, the frameworks and the expressiveness of the modelling languages. Most importantly the process model allows to identify the information used and produced during model creation. This information will lead to a consistent knowledge base of the enterprise in the course of enterprise modelling.

More work is still required on the contents of the different methodologies to establish its consistent process models. Work which can only be done by or in co-operation with the authors of the methodologies. For the comparison of the modelling languages the different constructs have to be compared on the attribute level to allow for thorough evaluation. Work which has only be done with CIMOSA and IEM[6]. Also identification of the information used and produced in the course of model creation is still far from complete. This identification has the potential of much more consistent modelling of enterprise information. An aspect which will increase the operational use of enterprise models considerably e.g. for decision support. If the knowledge base is kept consistent and up-to-date planning activities, evaluation of alternatives and investment decision will be based on current rather than historic information.

Additional benefits will be obtained by taking advantage of the common representation and converging terminology and task definitions. Today there is no common understanding on enterprise models and relating models from different enterprises is a rather difficult if not impossible task.

Even with the reasons accepted for the different methodologies, the need of compatibility remains for the user of enterprise modelling methodologies. Otherwise enterprise co-operation across organisation boundaries will not move into a really integrated mode and inter enterprise integration will never become a reality. A reality which is very much desirable for joint ventures and subcontractors or for their more modern versions of extended and virtual agile enterprises.

\section{REFERENCES}

[1] ESPRIT Consortium AMICE (1993), CIMOSA - Open System Architecture for CIM; Springer-Verlag, (ISBN 3-540-56256-7), (ISBN 0-387-56256-7).

[2] CIMOSA Association (1996), CIMOSA - Open System Architecture for CIM; Technical Baseline", Version 3.2, private publication.

[3] Williams, T. J. et al (1994) Architectures for integrating manufacturing activities and enterprises, Computers in Industry - 24, NR's 2-3, September.

[4] Mertins, et al (1991) Integrated Information Modelling for CIM: An Object-Oriented Method for Integrated Enterprise Modelling, Proceedings CAPE'91, North-Holland (ISBN 044489159 5).

[5] Scheer, A.-W. (1991) "Architektur integrierter Informationssysteme", Springer-Verlag, (ISBN 3-54053984-0) (in German).

[6] Vallespir, B. et. al (1991) Definition of a CIM Architecture within the ESPRIT IMPACS Project, Proceedings CAPE'91, North-Holland, (ISBN 044489159 5).

[7] Williams, T. J. (1994) The Purdue Enterprise Reference Architecture , Computers in Industry - Vol. 24, NR's. 2-3, September.

[8] Zelm, M. et al (1995) The CIMOSA Modelling Process, Computers in Industry, Vol. 27, NR. 2 October.

[9] Williams, T. J. (1995) The Generalised Reference Architecture and Methodology (GERAM) - a proposal from the IFAC/IFIP Task Force, in Ladet, P. and Vernadat, F.B. Integrated Manufacturing Systems Engineering, Chapman \& Hall (ISBN 0-412-72680-7)

[10] QCIM/WG QEM - ESPRIT CIMOSA (1994), Comparison CIMOSA - IEM Modelling Constructs and Methodology, CEN TC 310/WG1 Document N 41.3.

[11] ENV 12204 (1995) Advanced Manufacturing Technology - Systems Architecture - Constructs for Enterprise Modelling, CEN TC 310/WG1. 


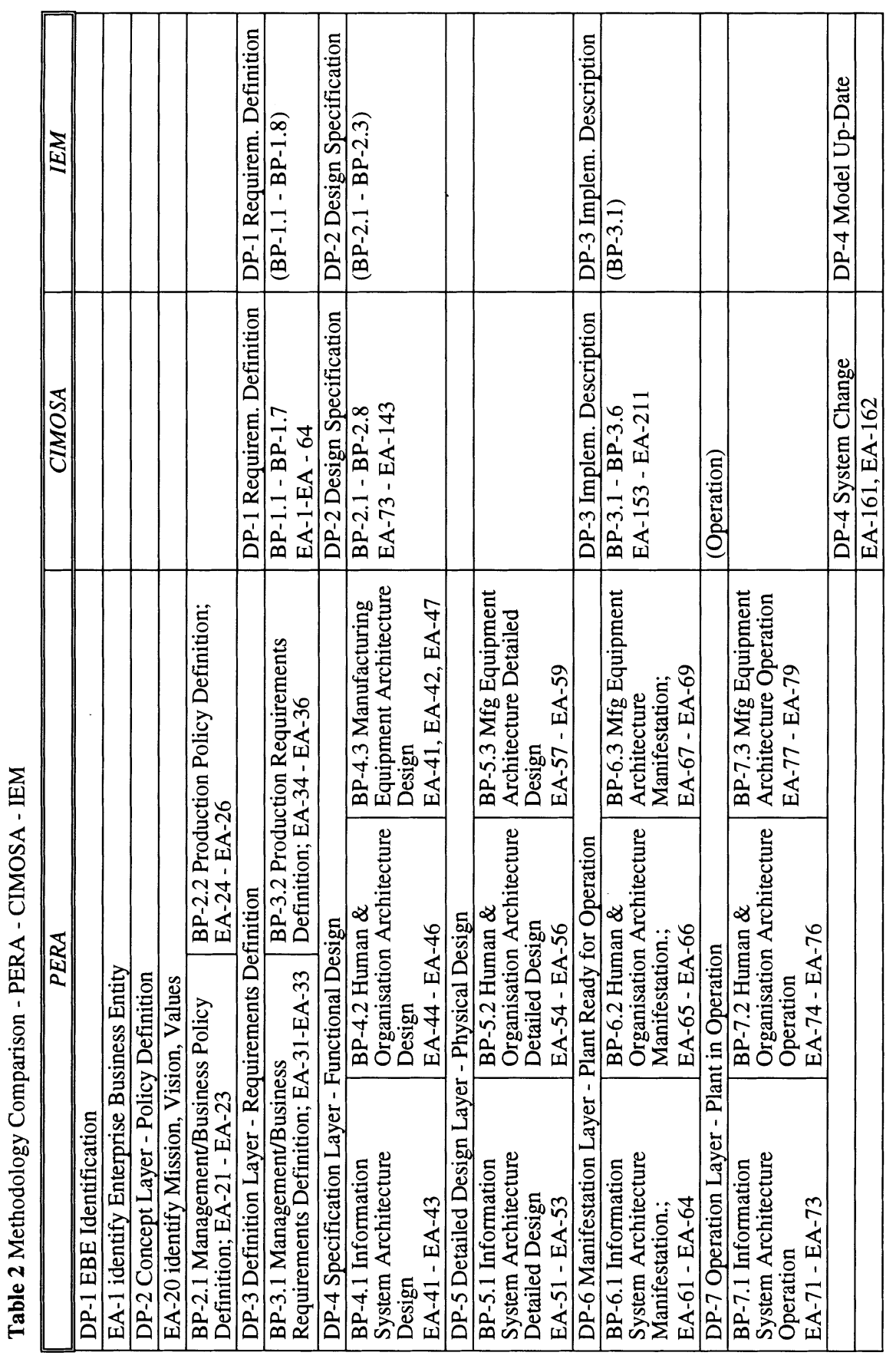



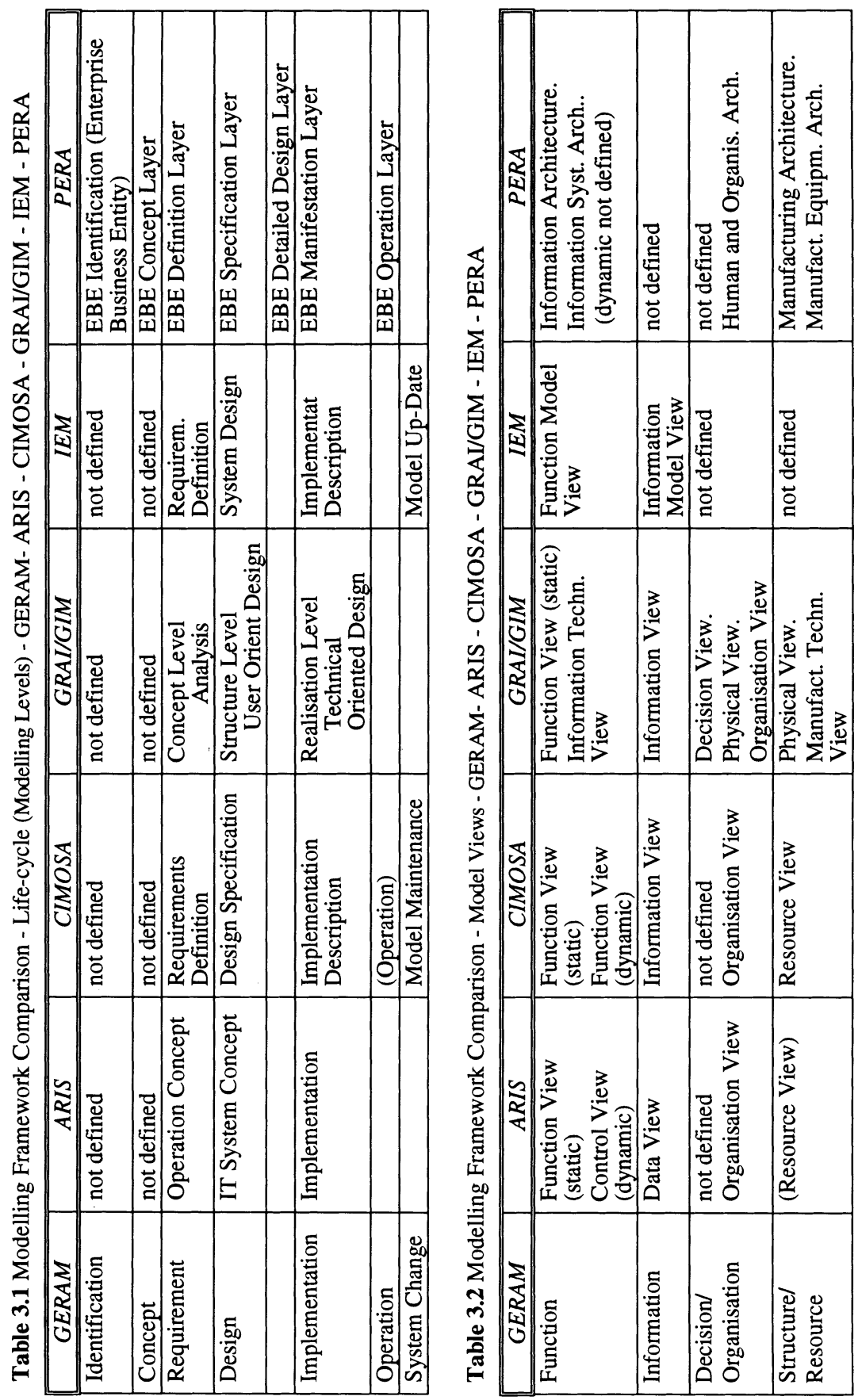


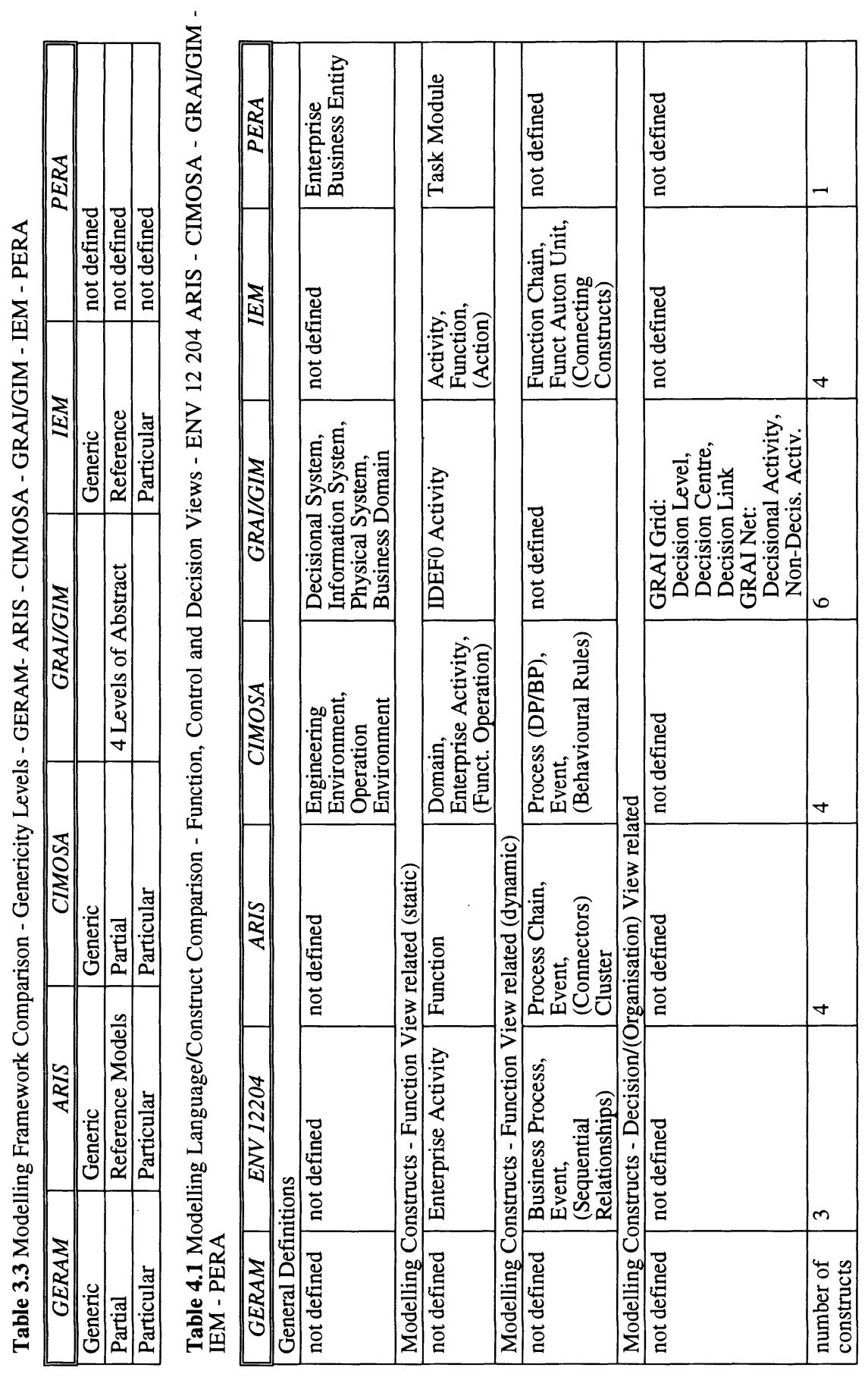




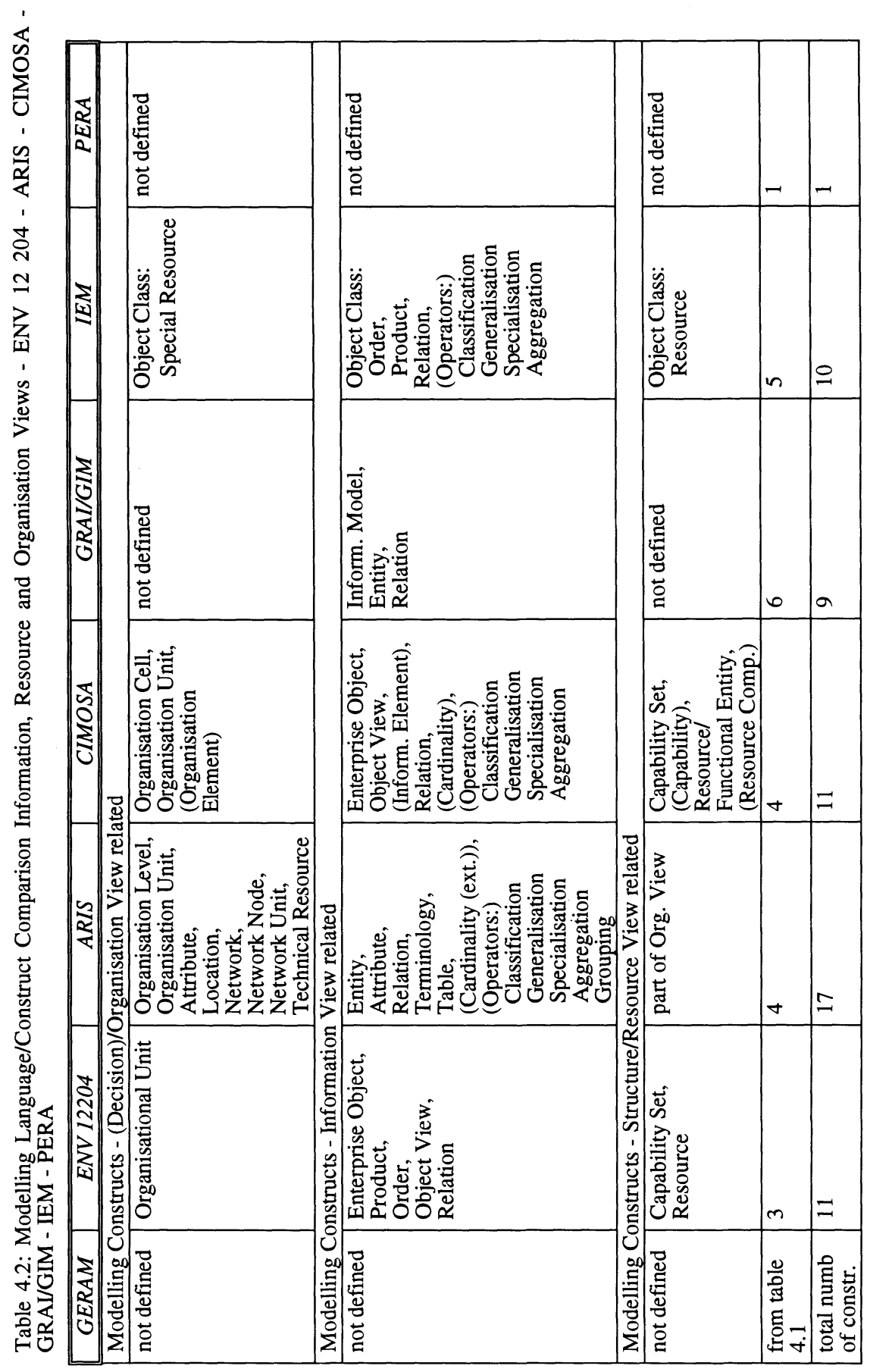

